December 5, 2018

\title{
Neutrino masses, mixing and leptogenesis in TeV scale $B-L$ extension of the standard model
}

\author{
M . Abbas and S. Khalil \\ Center for Theoretical Physics at the British University in Egypt, Sherouk City, Cairo \\ 11837, Egypt. \\ Faculty of Science, Ain Shams University, Cairo 11566, Egypt.
}

\begin{abstract}
We address the issue of the neutrino masses and mixing in $\mathrm{TeV}$ scale $B-L$ extension of the Standard Model. We show that if Dirac neutrino masses are of order $10^{-4} \mathrm{Gev}$, then the measured neutrino masses are correctly obtained. We propose a mass relation between quarks and leptons that may account for such small Dirac neutrino masses. We analyze the leptogenesis in this type of models and provide analytical expressions for the new contributions due to the predicted extra Higgs and extra neutral gauge boson. We find that thermal leptogenesis, with a resonant enhancement due to nearly degenerate right-handed neutrinos, can yield sufficient baryon asymmetry. Finally, we comment on a possible scheme for nonthermal leptogenesis, which is due to the decay of extra Higgs into right-handed neutrino.
\end{abstract}

\section{Introduction}

The Standard Model (SM) of electroweak and strong interactions has had a tremendous success when confronted with experiment. However, non-vanishing neutrino masses provides the first confirmed hint towards physics beyond the SM. The evidence of very light neutrino masses is now well established by measuring neutrino oscillations in solar and atmospheric neutrinos. It was shown that the minimal extension of the SM gauge group by an extra $U(1)$ gauge symmetry has all the necessary requirements to generate the observed neutrino masses [1]. In particular, this type of models has three SM singlet fermions that arise as a result of the anomaly cancellation conditions. These particles account for right-handed neutrinos and give a natural explanation for the seesaw mechanism.

Recently, a TeV scale $B-L$ symmetry breaking, based on the gauge group $G_{B-L} \equiv$ $S U(3)_{C} \times S U(2)_{L} \times U(1)_{Y} \times U(1)_{B-L}$, has been studied [2]. It was emphasized that this model can account for the current experimental results of the light neutrino masses and their large mixing. In addition, it predicts an extra SM singlet scalar (extra Higgs) and 
an extra neutral gauge boson corresponding to $B-L$ gauge symmetry [2]. These new particles may have significant impact on the SM phenomenology, hence lead to interesting signatures at Large Hadron Collider (LHC) [3].

On the other hand, the observed baryon asymmetry in the universe provides another indication for physics beyond the SM since it has been established that the strength of the $\mathrm{CP}$ violation in the $\mathrm{SM}$ is not sufficient to generate this asymmetry [4]. The CP violating decay of the right handed neutrinos may generate leptogenesis which is the most attractive mechanism to interpret this baryon asymmetry. Since the evidence of non-vanishing neutrino masses, there has been a growing interest concerning leptogenesis and it becomes a competent to the other baryogenesis mechanisms [5]. It is interesting to note that a possible solution to the two major problems, which represent the most serious evidences for new physics, can be naturally emerged in such simple extension of the SM.

The aim of this paper is to explore the issue of the neutrino masses, mixing, and leptogenesis in this type of low scale $B-L$ extension of the SM. We show that a low scale $B-L$ symmetry braking assists to find a mass relation between quarks and leptons, which may arise from a flavor symmetry. In this case, one can determine the unknown Dirac neutrino mass matrix, which is an essential for evaluating the lepton asymmetry. A detailed investigation for the leptogenesis in this type of models will be provided. We compute the new one-loop contributions to the decay of the lightest right handed neutrino due to the new extra Higgs and extra gauge boson.

Since these contributions do not include any strong CP violating phase, they do not interfere with the tree level contribution and hence they have no direct impact on the CP asymmetry. However, due to the fact that their size can be of the same order as the tree level one, their interference with the SM loop contributions can be relevant and enhance significantly the asymmetry. If the $B-L$ effect is negligible respect to the tree level, then the thermal leptogenesis is viable only if the right-handed neutrinos are nearly degenerate in mass so that the lepton asymmetry is resonantly enhanced. We show that $B-L$ contribution of order the tree level contribution can relax this degeneracy constraint. The non-thermal leptogenes is another interesting possibility for enhancing the lepton asymmetry. In this case, the lightest right handed neutrino can be decay product of heavier particle like, for instance, the $B-L$ extra Higgs or extra gauge boson. We show that this is a feasible scenario and is likely to take place in our model. However the out of equilibrium condition impose strong constrain on the mass of the heavy particle and on its coupling with right-handed neutrino. Therefore, the baryon asymmetry in non-thermal leptogenesis can be enhanced by two order of magnitude at most than that of thermal scenario.

The paper is organized as follows. In section 2 we briefly discuss the $B-L$ symmetry breaking and explore the possible constraints on the corresponding scale. Section 3 is devoted for neutrino masses and mixing in our TeV scale $B-L$ extension of the SM. We show that light neutrino masses can be generated through the seesaw mechanism if the Dirac neutrino masses are of order $10^{-4} \mathrm{GeV}$. This range of Dirac neutrino mass 
is consistent with possible relations may be obtained between the observed quark and lepton masses. In section 4 we investigate the lepton asymmetry due to the decay of lightest right-handed neutrino and analyze both scenarios of thermal and non-thermal leptogenesis. Finally we give our concluding remarks in section 5.

\section{$2 \quad B-L$ symmetry breaking}

We start our analysis by considering different scenarios of $B-L$ symmetry breaking. The relevant part for the Lagrangian of the leptonic sector in the minimal extension of the SM $S U(3)_{C} \times S U(2)_{L} \times U(1)_{Y} \times U(1)_{B-L}$ is given by

$$
\begin{aligned}
\mathcal{L}_{B-L} & =-\frac{1}{4} C_{\mu \nu} C^{\mu \nu}+i \bar{l} D_{\mu} \gamma^{\mu} l+i \bar{e}_{R} D_{\mu} \gamma^{\mu} e_{R}+i \bar{\nu}_{R} D_{\mu} \gamma^{\mu} \nu_{R}+\left(D^{\mu} \phi\right)^{\dagger}\left(D_{\mu} \phi\right) \\
& +\left(D^{\mu} \chi\right)^{\dagger}\left(D_{\mu} \chi\right)-V(\phi, \chi)-\left(\lambda_{e} \bar{l} \phi e_{R}+\lambda_{\nu} \bar{l} \tilde{\phi} \nu_{R}+\frac{1}{2} \lambda_{\nu_{R}} \overline{\nu_{R}^{c}} \chi \nu_{R}+\lambda_{\nu} \overline{\nu_{R}^{c}} \tilde{\phi} l^{c}+\text { h.c. }\right),
\end{aligned}
$$

where $C_{\mu \nu}=\partial_{\mu} C_{\nu}-\partial_{\nu} C_{\mu}$ is the field strength of the $U(1)_{B-L}$. The covariant derivative $D_{\mu}$ is generalized by adding the term $i g^{\prime \prime} Y_{B-L} C_{\mu}$, where $g^{\prime \prime}$ is the $U(1)_{B-L}$ gauge coupling constant and $Y_{B-L}$ is the $B-L$ quantum numbers of involved particles. The $Y_{B-L}$ for leptons and Higgs are given by: $Y_{B-L}(l)=-1, Y_{B-L}\left(e_{R}\right)=-1, Y_{B-L}\left(\nu_{R}\right)=-1$, $Y_{B-L}(\phi)=0$ and $Y_{B-L}(\chi)=2$. In Eq. $(1), \lambda_{e}, \lambda_{\nu}$ and $\lambda_{\nu_{R}}$ refer to $3 \times 3$ Yakawa matrices.

In order to analyze the $B-L$ and electroweak symmetry breaking, we consider the most general Higgs potential invariant under these symmetries, which is given by

$$
V(\phi, \chi)=m_{1}^{2} \phi^{\dagger} \phi+m_{2}^{2} \chi^{\dagger} \chi+\lambda_{1}\left(\phi^{\dagger} \phi\right)^{2}+\lambda_{2}\left(\chi^{\dagger} \chi\right)^{2}+\lambda_{3}\left(\chi^{\dagger} \chi\right)\left(\phi^{\dagger} \phi\right),
$$

where $\lambda_{3}>-2 \sqrt{\lambda_{1} \lambda_{2}}$ and $\lambda_{1}, \lambda_{2} \geq 0$, so that the potential is bounded from below. This is the stability condition of the potential. Furthermore, in order to avoid vanishing vacuum expectation values (vevs): $v=\langle\phi\rangle=0$ and $v^{\prime}=\langle\chi\rangle=0$ from being local minimum, one must assume that $\lambda_{3}^{2}<4 \lambda_{1} \lambda_{2}$. As in the usual Higgs mechanism of electroweak symmetry breaking in the SM, the $B-L$ spontaneous symmetry breaking requires a negative squared masse, $m_{2}^{2}<0$. In this case, the following non-zero vev may be obtained

$$
v^{\prime 2}=\frac{-2\left(m_{1}^{2}+\lambda_{1} v^{2}\right)}{\lambda_{3}},
$$

with

$$
v^{2}=\frac{4 \lambda_{2} m_{1}^{2}-2 \lambda_{3} m_{2}^{2}}{\lambda_{3}^{2}-4 \lambda_{1} \lambda_{2}} .
$$

From these equations, two comments are in order: $(i)$ For non-vanishing $\lambda_{3}$, the vevs $v$ and $v^{\prime}$ are related and hence they are naturally of the same order, i.e., $v \simeq \mathcal{O}(100) \mathrm{GeV}$ and $v^{\prime} \simeq \mathcal{O}(1) \mathrm{TeV}$. In fact, in this scenario $v^{\prime} \gg v$ will require a significant fine-tuning among the input parameters: $m_{1,2}^{2}$ and $\lambda_{1,2,3}$. (ii) The condition of the electroweak symmetry breaking, for $\lambda_{3}^{2}-4 \lambda_{1} \lambda_{2}<0$, is given by

$$
m_{1}^{2}<M_{C}^{2}=\frac{\lambda_{3} m_{2}^{2}}{2 \lambda_{2}} .
$$


For $m_{2}^{2}<0$ and $m_{1}^{2}>M_{C}^{2}, U(1)_{B-L}$ is spontaneously broken while the $S U(2)_{L} \times U(1)_{Y}$ remains exact. At this stage, the following vevs are obtained

$$
\begin{aligned}
v^{\prime} & =\sqrt{\frac{-m_{2}^{2}}{2 \lambda_{2}}}, \\
v & =0 .
\end{aligned}
$$

The evolution from $\mathcal{O}(1) \mathrm{TeV}$ scale down to $\mathcal{O}(100) \mathrm{GeV}$, may reduce the squared Higgs mass $m_{1}^{2}$ until eventually the minimization condition is satisfied and the electroweak gauge symmetry is broken. This scenario corresponds to two stages symmetry breaking at two different scales. Note that if $\lambda_{3}<0$, the radiative electroweak symmetry breaking can be achived with positive squared Higgs mass. Therefore, throughout this work, we will focus on the following region of mixing coupling $\lambda_{3}: 0>\lambda_{3}>-2 \sqrt{\lambda_{1} \lambda_{2}}$ and $\lambda_{1,2} \sim O(1)$.

As usual, we expand the scalar field $\chi$ around the $B-L$ minimum $v^{\prime}$ and write

$$
\chi(x)=\frac{v^{\prime}+H^{\prime}(x)}{\sqrt{2}} .
$$

In this case, one finds the following lagrangian for the $B-L$ Higgs $\left(H^{\prime}\right)$ mass and its interaction with right handed neutrino and SM Higgs $\phi$ :

$$
\mathcal{L}\left(\phi, H^{\prime}\right)=\frac{1}{2} m_{H^{\prime}}^{2} H^{\prime 2}-\frac{1}{2 \sqrt{2}} \lambda_{\nu_{R}} H^{\prime} \bar{\nu}_{R}^{c} \nu_{R}+\lambda_{3}\left(\frac{1}{2} H^{\prime 2} \phi^{2}+v^{\prime} H^{\prime} \phi^{2}\right) .
$$

Finally, after the $B-L$ gauge symmetry breaking the gauge field $C_{\mu}$ (will be called $Z^{\prime}$ in the rest of the paper) acquires the following mass:

$$
M_{Z^{\prime}}^{2}=4 g^{\prime \prime 2} v^{\prime 2} .
$$

The Lagrangian terms that describe the interactions of the $Z_{\mu}^{\prime}$ gauge boson are given by

$$
\mathcal{L}_{Z^{\prime}}=-\frac{1}{4} Z_{\mu \nu}^{\prime} Z^{\mu \nu}+\frac{1}{2} M_{Z^{\prime}}^{2} Z_{\mu}^{\prime} Z^{\mu}+2 g^{\prime \prime 2} Z_{\mu}^{\prime} Z^{\mu} \chi^{2}+4 g^{\prime \prime 2} v^{\prime} Z_{\mu}^{\prime} Z^{\prime \mu} \chi-i g^{\prime \prime} J_{\mu}^{B-L} Z^{\prime \mu}
$$

where $J_{\mu}^{B-L}=\bar{\psi}_{L} \gamma_{\mu} \psi_{L}+\bar{e}_{R} \gamma_{\mu} e_{R}+\bar{\nu}_{R} \gamma^{\mu} \nu_{R}$. The high energy experimental searches for an extra neutral gauge boson impose lower bounds on the $Z^{\prime}$ mass. The LEP II provides the most stringent constraint [6]. As $e^{+} e^{-}$collider, it was able to strongly constrain the extra-gauge boson that coupled significantly with electrons. It is worth noting that in this class of model, with the above particle assignments, any mixing effects between $U(1)$ factors may arise only at the two loop level, hence keeping them small enough. However, the measurements of $e^{+} e^{-} \rightarrow f \bar{f}$ above the $Z$-pole at LEP II impose stringent constraints on the mass of $Z_{B-L}$ or on the $B-L$ gauge coupling. Furthermore, the recent results by CDF II [7] are consistent with the LEP II constraints on $Z^{\prime}$ mass in case of $B-L$ extension of the SM. Therefore, the typical lower bound on $M_{Z^{\prime}}$ is now given by

$$
M_{Z^{\prime}} / g^{\prime \prime}>6 \mathrm{TeV} .
$$

Thus, one finds that $v^{\prime} \gtrsim O(\mathrm{TeV})$. 


\section{Neutrino masses and mixing in $B-L$ extension of the SM}

In this section we provide a detail analysis for the neutrino masses and mixing in the gauge $B-L$ extension of the SM, where the neutrino masses may be generated through a $\mathrm{TeV}$ scale seesaw mechanism. After $U(1)_{B-L}$ symmetry breaking [2], the Yukawa interaction term: $\lambda_{\nu_{R}} \chi \bar{\nu}_{R}^{c} \nu_{R}$ leads, as usual, to right handed neutrino mass: $M_{R}=\frac{1}{2 \sqrt{2}} \lambda_{\nu_{R}} v^{\prime}$. Also the electroweak symmetry breaking results in the Dirac neutrino mass term : $m_{D}=\frac{1}{\sqrt{2}} \lambda_{\nu} v$. Therefore, the mass matrix of the left and right-handed neutrinos is given by

$$
\left(\begin{array}{cc}
0 & m_{D} \\
m_{D} & M_{R}
\end{array}\right) .
$$

Since $M_{R}$ is proportional to $v^{\prime}$ and $m_{D}$ is proportional to $v$ i.e., $M_{R}>m_{D}$, the diagonalization of this mass matrix leads to the following masses for the light and heavy neutrinos respectively:

$$
\begin{aligned}
& m_{\nu_{L}}=-m_{D} M_{R}^{-1} m_{D}^{T}, \\
& m_{\nu_{H}}=M_{R} .
\end{aligned}
$$

Thus, $B-L$ gauge symmetry can provide a natural framework for the seesaw mechanism. However, the scale of $B-L$ symmetry breaking $v^{\prime}$ remains arbitrary. As in Ref.[2], $v^{\prime}$ is assumed to be of order TeV. Therefore, the value of $M_{R}$ is also of that order.

In our analysis, we adopt a basis where the charged lepton mass matrix and the Majorana mass matrix $M_{R}$ are both real and diagonal. Therefore, one can parameterize $M_{R}$ as follows

$$
M_{R}=M_{R_{3}}\left(\begin{array}{ccc}
r_{1} & 0 & 0 \\
0 & r_{2} & 0 \\
0 & 0 & 1
\end{array}\right) \text {, }
$$

where

$$
M_{R_{3}}=\left|\lambda_{\nu_{R_{3}}}\right| \frac{v^{\prime}}{2 \sqrt{2}}
$$

and

$$
r_{1,2}=\frac{M_{R_{1,2}}}{M_{R_{3}}}=\left|\frac{\lambda_{\nu_{R_{1,2}}}}{\lambda_{\nu_{R_{3}}}}\right| .
$$

As can be seen from Eq.(15) that even if $v^{\prime}$ is fixed to be of order TeV, the absolute value of $M_{R}$ is still parameterized by three known parameters. On the other hand, the Dirac mass matrix (if it is real) is given in terms of 9 parameters. Since $U(1)_{B-L}$ can not impose any further constraint to reduce the number of these parameters, the total number of free parameters involved in the light neutrino mass matrix are 12 parameters. As is known, the solar and atmospheric neutrino oscillation experiments have provided measurements for the neutrino mass-squared differences and also for the neutrino mixing angles. At the 
$3 \sigma$ level, the allowed ranges are [8] :

$$
\begin{aligned}
\Delta m_{12}^{2} & =(7.9 \pm 0.4) \times 10^{-5} \mathrm{eV}^{2} \\
\left|\Delta m_{32}^{2}\right| & =(2.4+0.3) \times 10^{-3} \mathrm{eV}^{2} \\
\theta_{12} & =33.9^{\circ} \pm 1.6^{\circ}, \\
\theta_{23} & =45^{\circ}, \\
\sin ^{2} \theta_{13} & \leq 0.048 .
\end{aligned}
$$

Therefore, the number of the experimental inputs are at most six: three neutrino masses (assuming possible ansatze like hierarchy or degenerate) and three mixing angles (if we assume $\left.\theta_{13}=0\right)$.

One of the interesting parametrization for the Dirac neutrino mass matrix is given by

$$
m_{D}=U_{M N S} \sqrt{m_{\nu}^{\text {diag }}} R \sqrt{M_{R}}
$$

where $m_{\nu}^{\text {diag }}$ is the physical neutrino mass matrix and $U_{M N S}$ is the lepton mixing matrix. The matrix $\mathrm{R}$ is an arbitrary orthogonal matrix which can be parameterized, in case of real $m_{D}$, in terms of three angles. In Eq.(19), the six unknown parameters are now given in terms of three masses in $M_{R}$ and the three angles in $R$. In order to fix these angles, one needs a flavor symmetry beyond the gauge symmetry which is typically flavor blind. Several types of flavor symmetries have been discussed in the literatures [9]. Here we follow different approach. We attempt to extend the observed relations between the masses of up quarks and charged leptons to the down quark and neutrino masses.

From the measured values of the up quark and charged lepton masses at the electroweak scale, one can notice the following relations

$$
\frac{m_{u}}{m_{c}} \sim \frac{m_{e}}{m_{\mu}} \sim O\left(10^{-3}\right),
$$

and

$$
\frac{m_{c}}{m_{t}} \sim \frac{m_{\mu}^{2}}{m_{\tau}^{2}} \sim O\left(10^{-3}\right)
$$

In the event of a flavor discrete-symmetry that may explain these ratios, the down quark and neutrino sectors may also be subjected to this symmetry. Hence, a similar relation may be obtained among their masses. If the scale of this discrete symmetry $\left(v_{F}\right)$ is below seesaw ( $B-L$ symmetry breaking) scale, then the above mass ratio would be extended to the down quark and light neutrino masses. In this case, one would expect that

$$
\begin{aligned}
& \frac{m_{d}}{m_{s}} \sim \frac{m_{\nu_{1}}}{m_{\nu_{2}}} \sim O\left(10^{-2}\right) \\
& \frac{m_{s}}{m_{b}} \sim \frac{m_{\nu_{2}}^{2}}{m_{\nu_{3}}^{2}} \sim O\left(10^{-2}\right) .
\end{aligned}
$$


However, if the scale of the flavor discrete-symmetry is above the seesaw mechanism scale, then the mass ration is anticipated to be between down quark and Dirac neutrino masses, i.e.,

$$
\begin{aligned}
& \frac{m_{d}}{m_{s}} \sim \frac{m_{D_{1}}}{m_{D_{2}}} \sim O\left(10^{-2}\right) \\
& \frac{m_{s}}{m_{b}} \sim \frac{m_{D_{2}}^{2}}{m_{D_{3}}^{2}} \sim O\left(10^{-2}\right)
\end{aligned}
$$

Let us start by considering the first scenario where $v_{F}<v^{\prime}$. The experimental results for the light neutrino masses in Eq.(18) leads to

$$
\begin{aligned}
& m_{\nu_{2}}=\sqrt{7.9 \times 10^{-5}-m_{\nu_{1}}^{2}}, \\
& m_{\nu_{3}}=\sqrt{\left|2.4 \times 10^{-3}-7.9 \times 10^{-5}+m_{\nu_{1}}^{2}\right|},
\end{aligned}
$$

with arbitrary $m_{\nu_{1}}$. Thus, for $m_{\nu_{1}}^{2} \ll 7.9 \times 10^{-5}$, the ansatz of hierarchal light neutrino masses is obtained. It is interesting to note that if $m_{\nu_{1}} \sim 10^{-4}$, the hierarchal ansatz is consistent with the mass relations given in Eqs. $(22,23)$ and the light neutrino mass matrix takes the form

$$
m_{\nu} \simeq 0.05 \mathrm{eV}\left(\begin{array}{ccc}
10^{-3} & 0 & 0 \\
0 & 0.16 & 0 \\
0 & 0 & 1
\end{array}\right)
$$

The Dirac neutrino mass matrix is now given by (for $r_{1} \sim r_{2} \sim 0.1, M_{R_{3}}=5 \mathrm{TeV}$, and order one angles/phases of $R$-matrix):

$$
m_{D} \simeq 10^{-3}\left(\begin{array}{ccc}
0.16+0.23 i & -0.25+0.16 i & -0.19-0.26 i \\
-0.22-0.34 i & 0.37-0.24 i & 0.30+0.38 i \\
-0.14+0.47 i & -0.53-0.15 i & 0.16-0.68 i
\end{array}\right)
$$

Note that the complex phases in $m_{D}$ are induced by the phases of $R$ matrix since the mixing matrix $U_{M N S}$ is real $\left(\theta_{13}=0\right.$ is assumed). These phases are crucial for generating lepton asymmetry as will be discussed in the next section. Also, as can be seen from the above example, $m_{D} \lesssim \mathcal{O}\left(10^{-4}\right) \mathrm{GeV}$, i.e., the Dirac neutrino Yukawa coupling $\lambda_{\nu}$ is of order $10^{-6}$, which is just one order of magnitude smaller than the electron Yukawa coupling.

Now we turn to the case of $v_{F}>v^{\prime}$. From Eqs. $(24,25)$, one gets

$$
m_{D}^{\text {diag }} \simeq m_{D_{3}}\left(\begin{array}{ccc}
10^{-3} & 0 & 0 \\
0 & 10^{-1} & 0 \\
0 & 0 & 1
\end{array}\right)
$$

If we assume hierarchal neutrino masses $m_{\nu_{1}} \ll m_{\nu_{2}} \ll m_{\nu_{3}}$, the light neutrino mass matrix can be written as

$$
m_{\nu} \simeq 0.05 \mathrm{eV}\left(\begin{array}{ccc}
m_{\nu_{1}} & 0 & 0 \\
0 & 0.16 & 0 \\
0 & 0 & 1
\end{array}\right)
$$


By using the determinant of $m_{D}$ from Eqs.(19) and (30), one can express $m_{D_{3}}$ in terms of $m_{\nu_{1}}, r_{1}, r_{2}$ and $M_{R_{3}}$ as follows:

$$
\left(\frac{m_{D_{3}}}{\mathrm{GeV}}\right) \simeq 10^{-4}\left(\frac{M_{R_{3}}}{\mathrm{GeV}}\right)^{1 / 2}\left[r_{1} r_{2}\left(\frac{m_{\nu_{1}}}{\mathrm{eV}}\right)\right]^{1 / 6}
$$

Here, we have used the fact that the determinant of the orthogonal matrix $R$ is one. Using this relation, one can determine, in terms of $M_{R_{3}}, r_{1}$ and $r_{2}$, the three angles $\left(\theta_{12}, \theta_{23}, \theta_{13}\right)$ that parameterize the matrix $R$ and lead to eigenvalues for the Dirac mass matrix $m_{D}$ consistent with our inputs in Eq.(30).

In case of $r_{1} \lesssim r_{2} \lesssim 1$ (hierarchy heavy neutrino masses), one finds that there is a possible solution for the angles $\theta_{i j}$ only for $m_{\nu_{1}}<10^{-7} \mathrm{GeV}$. In addition the angle $\theta_{13}$ can be fixed at $\theta_{13} \simeq \pi / 2$, hence the matrix $R$ is given by

$$
R=\left(\begin{array}{ccc}
0 & 0 & 1 \\
-\sin \alpha & \cos \alpha & 0 \\
-\cos \alpha & -\sin \alpha & 0
\end{array}\right),
$$

where $\alpha=\theta_{12}+\theta_{23}$. For instance, with $M_{R_{3}}=5 \mathrm{TeV}, r_{1} \simeq 0.1$ and $r_{2} \simeq 0.4$, one gets $\alpha \simeq 0.65$. Thus, the following $R$ matrix is obtained

$$
R=\left(\begin{array}{ccc}
0 & 0 & 1 \\
-0.6 & 0.8 & 0 \\
-0.8 & -0.6 & 0
\end{array}\right)
$$

While, for $r_{1} \simeq r_{2} \simeq 1$ (degenerate heavy neutrino masses), the matrix $R$ is given by

$$
R=\left(\begin{array}{ccc}
0 & 0 & 1 \\
-0.73 & 0.67 & 0 \\
-0.67 & -0.73 & 0
\end{array}\right)
$$

Finally, we can also have a complex $\mathrm{R}$, which induce a new source of $\mathrm{CP}$ violation phase in the Dirac Yukawa matrix $Y_{D}$. In this case, the angle $\alpha$ would be written as $\alpha=\rho+i \sigma$. For the above example of $r_{1}=0.1$ and $r_{2}=0.4$, the corresponding complex $R$-matrix is given by

$$
R=\left(\begin{array}{ccc}
0 & 0 & 1 \\
-0.6 e^{i a} & 0.8 e^{i a} & 0 \\
-0.8 e^{i a} & -0.6 e^{i a} & 0
\end{array}\right)
$$

It is important to mention that the complex phases in $R$ matrix are not related to any of the low energy phases, however, it plays a crucial role in leptogenesis .

Before closing this section, we comment on the scenario of degenerate light neutrino masses $\left(m_{\nu_{1}} \simeq m_{\nu_{2}} \simeq m_{\nu_{3}} \simeq \tilde{m}\right)$. From the astrophysical constraint: $\sum_{i} m_{\nu_{i}}<1 \mathrm{eV}$, one finds that $\tilde{m}<0.3 \mathrm{eV}$. In this case, one may write

$$
\begin{aligned}
& m_{\nu_{2}}=\sqrt{\tilde{m}^{2}+7.9 \times 10^{-5}}, \\
& m_{\nu_{3}}=\sqrt{\tilde{m}^{2}+2.4 \times 10^{-3}+7.9 \times 10^{-5}} .
\end{aligned}
$$


Therefore, the light neutrino mass matrix takes the form

$$
m_{\nu}=\tilde{m}\left(\begin{array}{ccc}
1 & & \\
& \sqrt{1+\frac{0.000079}{\tilde{m}^{2}}} & \\
& & \sqrt{1+\frac{0.002479}{\tilde{m}^{2}}}
\end{array}\right) \lesssim 0.3 \mathrm{eV}\left(\begin{array}{ccc}
1 & & \\
& 1.00044 & \\
& & 1.01368
\end{array}\right) .
$$

In this case, it is clear that the suggested mass relations between down type quark and neutrino masses should be implemented on the Dirac neutrino masses. However, we found that there is no any solution for the angles $\theta_{i j}$ that can account for $m_{D}$. Therefore, in our framework, the ansatz of degenerate neutrino masses is disfavored .

\section{TeV scale Leptogenesis in $B-L$ extension of the SM}

The recent observations indicate that the asymmetry between number density of baryon $\left(n_{B}\right)$ and of anti-baryon $\left(n_{\bar{B}}\right)$ of the universe is given by [10]

$$
Y_{B}=\frac{n_{B}-n_{\bar{B}}}{s}=\frac{n_{B}}{s}=(6.3 \pm 0.3) \times 10^{-10},
$$

where $s=2 \pi^{2} g_{*} T^{3} / 45$ is the entropy density and $g_{*}$ is the effective number of relativistic degrees of freedom.

As mentioned in the introduction, the possibility of originating this asymmetry through the $\mathrm{CP}$ violating decay of the heavy right-handed neutrino is an interesting mechanism known as Leptogenesis [5]. Within the framework of $B-L$ extension of the SM, the lepton asymmetry $\varepsilon_{i}$ is generated by the $\mathrm{CP}$ violating decays of $\nu_{R_{i}}$ into the Higgs doublet and the charged lepton doublet $l_{\alpha}$, i.e., $\nu_{R_{i}} \rightarrow \phi+l_{\alpha}$ where $\alpha=(e, \mu, \tau)$. The lepton asymmetry is usually dominated by the $\nu_{R_{1}}$ decay:

$$
\varepsilon_{1}=\frac{\sum_{\alpha}\left(\left|A\left(\nu_{R_{1}} \rightarrow \phi l_{\alpha}\right)\right|^{2}-\left|A\left(\nu_{R_{1}} \rightarrow \bar{\phi} \bar{l}_{\alpha}\right)\right|^{2}\right)}{\sum_{\alpha}\left(\left|A\left(\nu_{R_{1}} \rightarrow \phi l_{\alpha}\right)\right|^{2}+\left|A\left(\nu_{R_{1}} \rightarrow \bar{\phi} \bar{l}_{\alpha}\right)\right|^{2}\right)}
$$

where $A\left(\nu_{R_{1}} \rightarrow \phi l_{\alpha}\right)$ is the total (tree plus loop) decay amplitude. Similar to the SM extended by three right handed neutrinos, the decay of $\nu_{R_{1}}$ into $\phi$ and $l_{\alpha}$ may occur through the tree level diagram, one loop vertex correction, and one loop self-energy, as shown in Fig. 1. However, in $B-L$ extension of the SM the decay of the right-handed neutrino into Higgs and the lepton doublets can be also generated through diagrams mediated by extra Higgs and extra gauge boson exchange, as displayed in Fig. 2.

In order to analyze the $\mathrm{CP}$ asymmetry $\varepsilon_{1}$, one may parameterize the decay amplitude as $\mathrm{CP}$ violating part times a CP conserving part (comes mainly from the loop function). In this respect, one may write tree level $A_{0}\left(\nu_{R_{1}} \rightarrow \phi l_{\alpha}\right)$ and one loop level $A_{1}\left(\nu_{R_{1}} \rightarrow \phi l_{\alpha}\right)$ as follows:

$$
\begin{array}{ll}
A_{0}\left(\nu_{R_{1}} \rightarrow \phi l_{\alpha}\right)=A_{\text {tree }}, & \bar{A}_{0}\left(\nu_{R_{1}} \rightarrow \phi l_{\alpha}\right)=A_{\text {tree }}^{*} \\
A_{1}\left(\nu_{R_{1}} \rightarrow \phi l_{\alpha}\right)=A_{\text {loop }} \times F, & \bar{A}_{0}\left(\nu_{R_{1}} \rightarrow \phi l_{\alpha}\right)=A_{\text {loop }}^{*} \times F .
\end{array}
$$



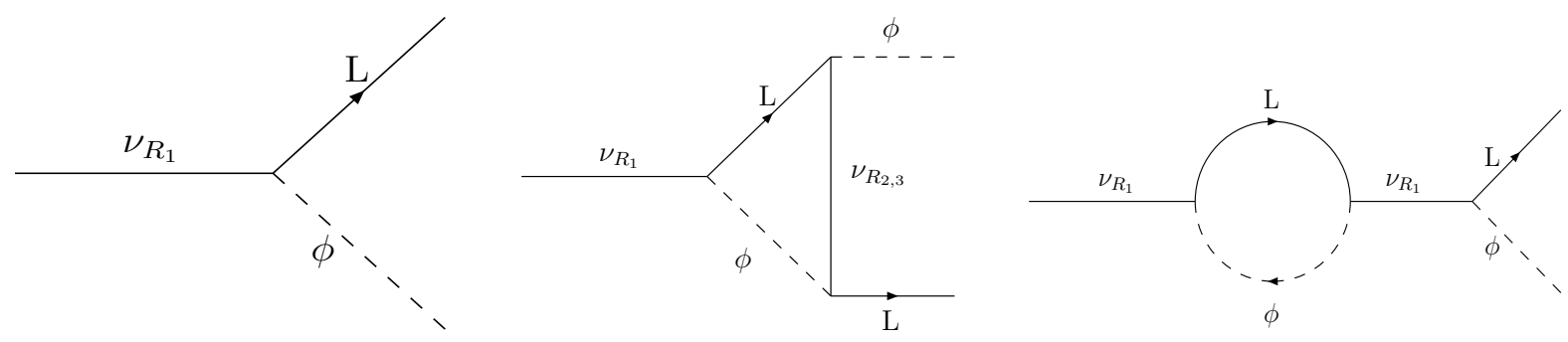

Figure 1: Feynman diagrams in SM with right-handed neutrinos that contribute to the decay $\nu_{R_{1}} \rightarrow \phi l_{\alpha}$.
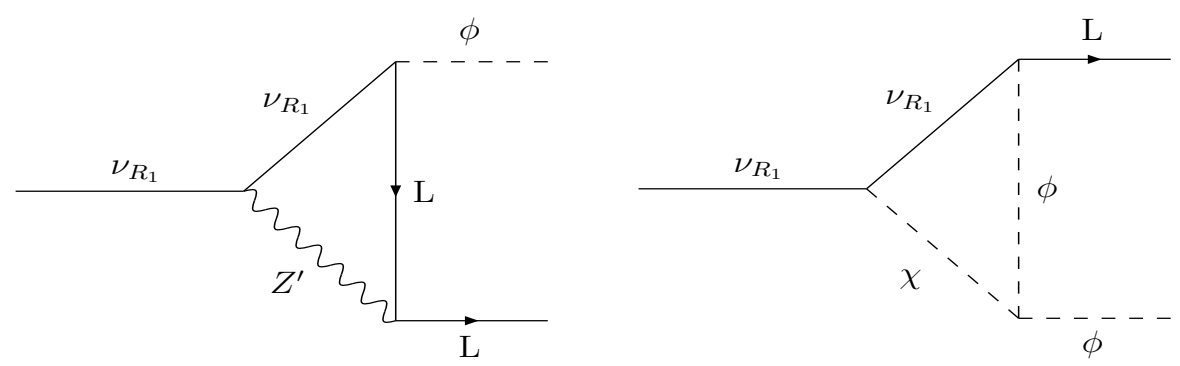

Figure 2: The new contributions to the decay $\nu_{R_{1}} \rightarrow \phi l_{\alpha}$ in $B-L$ extension of the SM.

The CP asymmetry arises through the interference between tree and loop contributions, hence $\varepsilon_{1}$ can be written as

$$
\varepsilon_{1}=\frac{\operatorname{Im}\left[A_{\text {tree }} A_{\text {loop }}^{*}\right] \operatorname{Im}[F]}{\left|A_{\text {tree }}\right|^{2}+\left|A_{\text {loop }} F\right|^{2}+2 \operatorname{Re}[F] \operatorname{Re}\left[A_{\text {tree }} A_{\text {loop }}^{*}\right]},
$$

Since $\left|A_{\text {tree }}\right| \gg\left|A_{\text {loop }} F\right|$, the CP asymmetry $\varepsilon_{1}$ is usually approximated as

$$
\varepsilon_{1} \simeq \frac{1}{\left|A_{\text {tree }}\right|^{2}} \operatorname{Im}\left[A_{\text {tree }} A_{\text {loop }}^{*}\right] \operatorname{Im}[F]
$$

The tree level contribution to the $\nu_{R_{1}}$ decay amplitude is given by

$$
A_{0}\left(\nu_{R_{1}} \rightarrow \phi l_{\alpha}\right)=-i\left(\lambda_{\nu}\right)_{\alpha 1}\left(\bar{u}(p) P_{R} u^{c}(q)\right)
$$

where $\bar{u}$ is the Dirac spinor of outgoing particle $l_{\alpha}$ with momentum $p$ and $u^{c}(q)$ is the spinor of the ingoing particle $\nu_{R_{1}}$ with momentum $q$. As can be seen from the above expression, the tree level contribution is proportional to Dirac neutrino Yukawa couplings $\left(\lambda_{\nu}\right)_{\alpha 1}$ which is of order $10^{-6}$, hence it is quite small. The contribution to the decay amplitude from the vertex correction is given by

$$
A_{V}\left(\nu_{R_{1}} \rightarrow \phi l_{\alpha}\right)=\frac{i}{16 \pi^{2}} \sum_{i}\left(\lambda_{\nu}\right)_{\alpha i}\left(\lambda_{\nu}^{\dagger} \lambda_{\nu}\right)_{1 i}\left(\bar{u}(p) P_{R} u^{c}(q)\right) F_{V}\left(\frac{M_{R_{i}}^{2}}{M_{R_{1}}^{2}}\right)
$$


The loop function $F_{V}(x)$ is given by

$$
\begin{aligned}
\operatorname{Re} F_{V}(x) & =-\sqrt{x}\left[1+\ln (x)\left[1-(1+x) \ln \left(\frac{1+x}{x}\right)\right],\right. \\
\operatorname{Im} F_{V}(x) & =\sqrt{x}\left[1-(1+x) \ln \left(\frac{1+x}{x}\right)\right] .
\end{aligned}
$$

The self-energy diagram leads to the following contribution to the decay amplitude of $\nu_{R_{1}} \rightarrow \phi l_{\alpha}$

$$
A_{S}\left(\nu_{R_{1}} \rightarrow \phi l_{\alpha}\right)=\frac{i}{16 \pi^{2}} \sum_{i}\left(\lambda_{\nu}\right)_{\alpha i}\left(\lambda_{\nu}^{\dagger} \lambda_{\nu}\right)_{1 i}\left(\bar{u}(p) P_{R} u^{c}(q)\right) F_{S}\left(\frac{M_{i}^{2}}{M_{R_{1}}^{2}}\right)
$$

where the corresponding loop function $F_{S}(x)$ is given by [11]

$$
F_{S}\left(\frac{M_{R_{i}}^{2}}{M_{R_{1}}^{2}}\right)=\left|\omega_{1 i}\left(M_{R_{1}}^{2}\right)\right|^{2}\left(M_{R_{1}}^{2}-M_{R_{i}}^{2}\right) \frac{M_{R_{i}}}{M_{R_{1}}}
$$

with

$$
\omega_{1 i}\left(M_{R_{1}}^{2}\right)^{-1}=\frac{\left(M_{R_{1}}^{2}-M_{R_{i}}^{2}\right)}{M_{R_{1}}}-2 a\left(M_{R_{1}}^{2}\right)\left(M_{R_{1}}\left(\lambda_{\nu}^{\dagger} \lambda_{\nu}\right)_{i i}-M_{R_{i}}\left(\lambda_{\nu}^{\dagger} \lambda_{\nu}\right)_{11}\right)
$$

and

$$
a\left(q^{2}\right)=\frac{1}{16 \pi^{2}}\left(\ln \frac{q^{2}}{\mu^{2}}-2-i \pi \Theta\left(q^{2}\right)\right) .
$$

It is clear that the loop function $F_{S}$ approaches zero in case of degenerate right-handed neutrino masses and hence the amplitude $A_{S}$ vanishes identically. In our model with $\mathrm{TeV}$ scale seesaw mechanism, the Dirac neutrino Yukawa couplings $\left(\lambda_{\nu}\right)_{i j}$ are of order $10^{-6}$. Therefore, the second term in $w_{1 i}^{-1}$ is much smaller than the first term, unless the righthanded neutrino masses are completely degenerate. In this respect, the loop function $F_{S}$ in Eq.(51) is reduced to

$$
F_{S}\left(\frac{M_{R_{i}}^{2}}{M_{R_{1}}^{2}}\right)=\frac{M_{R_{1}} M_{R_{i}}}{M_{R_{1}}^{2}-M_{R_{i}}^{2}}
$$

Now we turn to the new $B-L$ contributions to the right-handed neutrino decay due to the exchanges of extra $Z^{\prime}$ and $H^{\prime}$ as shown in Fig. 2. It is expected that these diagrams do not include strong CP phases since the running particles in the loops are heavier than initial particle $\nu_{R_{1}}$ and hence the these diagrams can not be cut in two parts. As a result, these diagrams will not directly contribute to the lepton asymmetry. However, These contributions may be of order the tree level and hence they affect the interference with vertex and self-energy corrections that remain the only sources of strong CP phases.

Our result for the extra Higgs contribution to the decay amplitude of $\nu_{R_{1}} \rightarrow \phi l_{\alpha}$ leads to

$$
A_{\chi}\left(\nu_{R_{1}} \rightarrow \phi l_{\alpha}\right)=\frac{1}{16 \pi^{2} M_{R_{1}}}\left(\lambda_{\nu_{R}}\right)_{11}\left(\lambda_{\nu}\right)_{1 \alpha} g_{\phi^{2} \chi}\left(\bar{u}(p) P_{R} u^{c}(q)\right) F_{\chi}\left(\frac{M_{\chi}^{2}}{M_{R_{1}}^{2}}\right)
$$


From Eq.(8), the coupling $g_{\phi^{2} \chi}$ is given by :

$$
g_{\phi^{2} \chi}=\sqrt{2} v^{\prime} \lambda_{3} .
$$

As can be seen from Eq.(2), the mixing parameter $\lambda_{3}$ is real thus the coupling $g_{\phi^{2} \chi}$ is real. Moreover, as explained in the previous section, the Yukawa coupling $\left(\lambda_{\nu_{R}}\right)_{11}$ is real too. In general it has the form:

$$
\left(\lambda_{\nu_{R}}\right)_{11}=\frac{2 \sqrt{2} M_{R_{1}}}{v^{\prime}} .
$$

Finally, $F_{\chi}(x)$ is the associate loop function which is given by

$$
\begin{aligned}
F_{\chi}(x) & =1-\frac{\pi^{2}}{6}-\left(\frac{x}{2}+\ln (2)-1\right) \ln (x)-\frac{1}{2} \sqrt{x(x-4)} \ln \left(\frac{x-\sqrt{x(x-4)}}{x+\sqrt{x(x-4)}}\right) \\
& -\ln (x-\sqrt{x(x-4)}) \ln \left(\frac{x-\sqrt{x(x-4)}-2}{x-\sqrt{x(x-4)}}\right) \\
& -\ln (x+\sqrt{x(x-4)}) \ln \left(\frac{x+\sqrt{x(x-4)}-2}{x+\sqrt{x(x-4)}}\right) \\
& +\operatorname{Li}_{2}\left(\frac{-x+\sqrt{x(x-4)}+2}{-x+\sqrt{x(x-4)}}\right)+\operatorname{Li}\left(\frac{x+\sqrt{x(x-4)}-2}{x+\sqrt{x(x-4)}}\right)
\end{aligned}
$$

Our computation for the the extra gauge boson $Z^{\prime}$ contribution to the decay amplitude of $\nu_{R_{1}} \rightarrow \phi l_{\alpha}$ leads to

$$
A_{Z^{\prime}}\left(\nu_{R_{1}} \rightarrow \phi l_{\alpha}\right)=\frac{1}{4 \pi^{2}} g^{\prime \prime 2}\left(\lambda_{\nu}\right)_{\alpha 1}\left(\bar{u}(p) P_{R} u^{c}(q)\right) F_{Z^{\prime}}\left(\frac{M_{z^{\prime}}^{2}}{M_{R_{1}}^{2}}\right),
$$

where $F_{Z^{\prime}}(x)$, at the $M_{R_{1}}$ scale, is given by

$$
\begin{aligned}
F_{Z^{\prime}}(x) & =-\frac{3}{2}+\frac{\pi^{2}}{12}-\sqrt{-x(x-4)}(x+1)\left[\tan ^{-1}\left(\frac{x-2}{\sqrt{-x(x-4)}}\right)+\tan ^{-1}\left(\frac{\sqrt{-x}}{\sqrt{(x-4)}}\right)\right] \\
& +\left(1+\frac{1}{4} x+x \ln (4)-\frac{1}{2}(1-4 x) \ln 2\right) \ln (x)-2 \pi^{2} x \\
& +-\left(\frac{1}{4}+x\right) \sqrt{x(x-4)} \ln \left(\frac{-\sqrt{x(x-4)}+x}{\sqrt{x(x-4)}+x}\right) \\
& +2 x \ln \left[\frac{x+\sqrt{x(x-4)}}{x}\right] \ln \left(\frac{-2+x-\sqrt{x(x-4)}}{x-\sqrt{x(x-4)}}\right) \\
& +2 x \ln \left[\frac{x-\sqrt{x(x-4)}}{x}\right] \ln \left(\frac{-2+x+\sqrt{x(x-4)}}{x+\sqrt{x(x-4)}}\right)
\end{aligned}
$$




$$
\begin{aligned}
& -\frac{1}{2}(1-4 x) \ln [x+\sqrt{x(x-4)}] \ln \left(\frac{-2+x+\sqrt{x(x-4)}}{x+\sqrt{x(x-4)}}\right) \\
& -\frac{1}{2}(1-4 x) \ln [x-\sqrt{x(x-4)}] \ln \left(\frac{-2+x-\sqrt{x(x-4)}}{x-\sqrt{x(x-4)}}\right) \\
& +\frac{1}{2} L i_{2}\left(\frac{1}{2}\left(1-\frac{\sqrt{(x-4)}}{\sqrt{x}}\right)\right)+\frac{1}{2} L i_{2}\left(\frac{1}{2}\left(1+\frac{\sqrt{(x-4)}}{\sqrt{x}}\right)\right)
\end{aligned}
$$

From the above expressions, it can be easily noted that the $\mathrm{CP}$ violating effect in the amplitudes $A_{V}\left(\nu_{R_{1}} \rightarrow \phi l_{\alpha}\right)$ and $A_{S}\left(\nu_{R_{1}} \rightarrow \phi l_{\alpha}\right)$ arises from the same source: $\left(\lambda_{\nu}\right)_{\alpha i}\left(\lambda_{\nu}^{+} \lambda_{\nu}\right)_{1 i}$. While in $A_{\chi}\left(\nu_{R_{1}} \rightarrow \phi l_{\alpha}\right)$ and $A_{Z^{\prime}}\left(\nu_{R_{1}} \rightarrow \phi l_{\alpha}\right)$ they are proportional to $g_{\phi^{2} \chi}\left(\lambda_{\nu_{R}}\right)_{11}\left(\lambda_{\nu}\right)_{1 \alpha}$ and $g^{\prime \prime 2}\left(\lambda_{\nu}\right)_{\alpha 1}$, respectively. Therefore, if $\lambda_{\nu} \sim \mathcal{O}\left(10^{-6}\right)$, as found in previous section, the $\chi$ and $Z^{\prime}$ may give significant contributions. However, due to the absence of strong CP violation in these processes, they have no interference with tree level diagram. Nevertheless, they may have significant effect through the interference with the SM one loop amplitudes $A_{V}$ and $A_{S}$. In this case, the total asymmetry is given by:

$$
\begin{aligned}
\varepsilon_{1} \simeq & \frac{1}{8 \pi\left(\lambda_{\nu}^{\dagger} \lambda_{\nu}\right)_{11}} \sum_{i=2,3}\left[\frac{\operatorname{Im}\left\{\left(\lambda_{\nu}^{\dagger} \lambda_{\nu}\right)_{1 i}^{2}\right\}}{\left(1-\frac{1}{4 \pi^{2}} g^{\prime \prime 2} F_{Z^{\prime}}\left(\frac{M_{z^{\prime}}^{2}}{M_{R_{1}}^{2}}\right)-\frac{1}{4 \pi^{2}} \lambda_{3} F_{\chi}\left(\frac{M_{\chi}^{2}}{M_{R_{1}}^{2}}\right)\right)}\right] \\
& {\left[\operatorname{Im} F_{V}\left(\frac{M_{R_{i}}^{2}}{M_{R_{1}}^{2}}\right)+\operatorname{Im} F_{S}\left(\frac{M_{R_{i}}^{2}}{M_{R_{1}}^{2}}\right)\right] . }
\end{aligned}
$$

If the new contributions due to $\chi$ and $Z^{\prime}$ exchanges have been neglected, one gets the usual CP asymmetry $\varepsilon_{1}$ of the SM extended by right handed neutrinos, which is given by

$$
\varepsilon_{1}^{\mathrm{SM}} \simeq \frac{1}{8 \pi} \frac{1}{\left(\lambda_{\nu}^{\dagger} \lambda_{\nu}\right)_{11}} \sum_{i=2,3} \operatorname{Im}\left\{\left(\lambda_{\nu}^{\dagger} \lambda_{\nu}\right)_{1 i}^{2}\right\}\left[\operatorname{Im} F_{V}\left(\frac{M_{R_{i}}^{2}}{M_{R_{1}}^{2}}\right)+\operatorname{Im} F_{S}\left(\frac{M_{R_{i}}^{2}}{M_{R_{1}}^{2}}\right)\right]
$$

From the equations, few comments are in order: $(i)$ The lepton asymmetry obtained in SM with right handed neutrinos is sensitive to the CP phase of $\left(\lambda_{\nu} \lambda_{\nu}^{\dagger}\right)$. Therefore, the necessary condition for the mechanism of leptogenesis to work is

$$
\operatorname{Im}\left(\lambda_{\nu}^{\dagger} \lambda_{\nu}\right)_{1 i} \neq 0 \Rightarrow \operatorname{Im}\left(\sqrt{M_{R}} R m_{\nu}^{\operatorname{diag}} R^{+} \sqrt{M_{R}}\right) \neq 0, i=2,3
$$

(ii) Due to the unitarity of the $U_{\text {MNS }}$, leptogenesis does not depend on the phases (if any) appearing in the leptonic mixing matrix. (iii) If the matrices $R$ and $M_{R}$ are real, then $\varepsilon_{1}=0$ and hence the leptogenesis vanishes identically. (vi) In the limit of quasidegenerate right-handed neutrinos i.e., $x=\left(M_{R_{2}} / M_{R_{1}}\right)^{2} \sim 1$, an enhancement for $\varepsilon_{1}$, due to the nearly vanishing of the denominator of $F_{S}(x)$, is obtained [12]. $(v)$ A possible enhancement for $\varepsilon_{1}$ can be achieved if $\frac{g^{\prime \prime}}{4 \pi} F_{Z^{\prime}} \simeq 1$. Note that since $\lambda_{3}<1$, the $H^{\prime}$ contribution is typically smaller than the tree level one. 


\subsection{Thermal leptogenesis}

This is the simplest scenario for leptogenesis where the lightest right-handed neutrino, $\nu_{R_{1}}$, is assumed to be in equilibrium while the heavier ones are decaying [13] . In this respect, the leptogenesis can be realized by the out of equilibrium of $\nu_{R_{1}}$ at temperature below its mass scale. To avoid washing out the asymmetry $\varepsilon_{1}$ by inverse decay and scattering processes, the total width of $\nu_{R_{1}}$ decay should be smaller than the expansion rate of the universe at temperature $T=M_{R_{1}}$. This is known as out-of-equilibrium condition, which implies that [13]

$$
Y_{L}=\frac{n_{L}-n_{\bar{L}}}{s}=\eta \frac{\varepsilon_{1}}{g_{*}}
$$

where $\eta$ is the efficiency factor which parameterizes the amount of washing out which depends on the size of $r=\Gamma_{1} / H\left(M_{R_{1}}\right) \simeq m_{\nu_{1}} / m^{*}$ where $m^{*}=256 \sqrt{g_{*}} v^{2} / 3 M_{P}$. If $r \ll 1$ i.e., $\nu_{R_{1}}$ decays strongly out-of-equilibrium, then $\eta \sim 1$. For $r \gg 1$, the lepton asymmetry is suppressed by $\eta \simeq 1 / r$. Finally. the electroweak sphaleron effects convert the lepton asymmetry $Y_{L}$ to baryon asymmetry $Y_{B}$ through a conversion factor $c[13]$ :

$$
Y_{B}=\frac{c}{c-1} Y_{L} \simeq-1.4 \times 10^{-3} \eta \varepsilon_{1}
$$

From this expression, it is clear that $Y_{B}$ can be of order the observed results reported

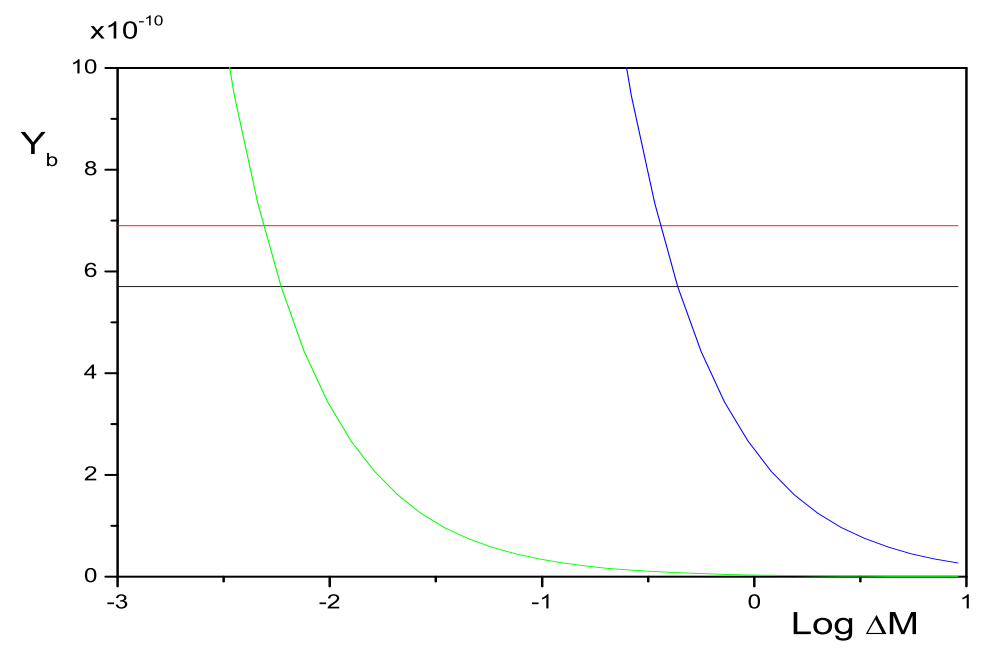

Figure 3: Baryon asymmetry in $B-L$ extension of the SM versus the mass difference of the first two right-handed neutrinos. Horizontal lines indicate the allowed $2 \sigma$ region of $Y_{B}$.

in Eq.(40) only if $\varepsilon_{1}$ is of order $\mathcal{O}\left(10^{-6}\right)$, assuming $\eta \sim 1$. However, as mentioned, unless the masses of the first two right-handed neutrinos are quite degenerate and/or the 
$Z^{\prime}$ contribution is of order the tree level one, the lepton asymmetry is a few order of magnitude below this value.

The baryon asymmetry as function of the mass difference $\Delta M=M_{R_{2}}-M_{R_{1}}$ is shown in Fig.3. As can be seen from this figure, In case of negligible $Z^{\prime}$ contribution, one needs $\Delta M \simeq \mathcal{O}\left(10^{-3}\right)$ to have $Y_{B}$ within the $2 \sigma$ range of the experimental measurements. While, for large $Z^{\prime}$ contribution, this degeneracy constrain is relaxed and mass difference of order $10 \%$ can account for the observed baryon asymmetry.

\subsection{Non-thermal leptogenesis}

Now we consider the possibility of having non-thermal leptogenesis [14]. As mentioned in the introduction, in the non-thermal leptogenesis scenario the right-handed neutrino is a decay product of a heavier particle. It is interesting to note that in our model of $B-L$, the extra Higgs $\chi$ and extra gauge boson $Z^{\prime}$ have direct couplings with the right handed neutrino $\nu_{R_{1}}$. In case, of extra-Higgs, this coupling is given (see Eq.8) by $-\lambda_{\nu_{R_{1}}} / 2 \sqrt{2}$. Therefore, the decay $\chi \rightarrow \nu_{R_{1}} \nu_{R_{1}}$ is kinematically allowed if $m_{\chi}>2 M_{R_{1}}$. In this case, one finds the following decay rate $\Gamma_{\chi}$ :

$$
\Gamma_{\chi}=\Gamma\left(\chi \rightarrow \nu_{R_{1}} \nu_{R_{1}}\right)=\frac{1}{4 \pi}\left|\lambda_{\chi \nu_{R_{1}}}\right|^{2} M_{\chi}=\frac{1}{4 \pi} \frac{M_{R_{1}}^{2}}{v^{\prime 2}} M_{\chi}
$$

The reheating temperature after this decay is given by

$$
T_{R}=\left(\frac{45}{4 \pi^{3} g_{*}}\right)^{1 / 4}\left(\Gamma_{\chi} M_{P}\right)^{1 / 2},
$$

where $M_{P}$ is Planck mass. In this framework, the lepton asymmetry is given by [14]

$$
Y_{L}=\frac{3}{2} B R\left(\chi \rightarrow \nu_{R_{1}} \nu_{R_{1}}\right) \frac{T_{R}}{M_{\chi}} \varepsilon_{1} .
$$

In order to avoid the inverse decay of $\chi \rightarrow \nu_{R_{1}} \nu_{R_{1}}$, the decay rate should be less than the universe expansion rate, i.e., $\Gamma\left(\chi \rightarrow \nu_{R_{1}} \nu_{R_{1}}\right) \lesssim H\left(\frac{M_{\chi}}{M_{P}}\right)$, where $H\left(M_{\chi}\right) \sim 1.7 \sqrt{g_{*}} M_{\chi}^{2} / M_{P}$ with $g_{*}=100$ in the SM. This out of equilibrium condition imposes stringent constrain on the coupling $\lambda_{\chi \nu_{R_{1}}}$ and/or on the extra-Higgs mass. For instance, if $M_{\chi} \simeq \mathcal{O}(100) \mathrm{TeV}$ then $\lambda_{\chi \nu_{R_{1}}}$ should be $\lesssim 10^{-5}$ and the associated reheating temperature is of order $\lesssim 10^{7}$ $\mathrm{GeV}$. Therefore, the factor $T_{R} / M_{\chi}$ is of order $10^{2}$. In this case, the the lepton asymmetry is enhanced by two order of magnitude at most. It is clear that this enhancement alone is not enough to account for the measured baryon asymmetry in the universe. However, it helps in relaxing the condition of nearly degenerate right-handed neutrino masses.

\section{Conclusions}

In this paper we have systematically analyzed the phenomenological implications for $\mathrm{TeV}$ scale $B-L$ extension of the SM. We have investigated the possible scenarios of symmetry breaking and the consequence on low energy experiments. We have studied the 
neutrino masses and mixing in this type of models. We have shown that the low scale seesaw mechanism is naturally implemented. However, to fix the free parameters of the neutrino sector and determine the Dirac neutrino mass matrix, a kind of flavor symmetry is required. We assumed a phenomenological mass relation between quarks and leptons. In this respect, we found that a hierarchal ansatz for light neutrino masses is favored.

We have also analyzed the leptogenesis in this class of models. We computed the new contributions to the $\mathrm{CP}$ violating decay of right handed neutrino to Higgs and leptons, due to the extra Higgs and extra gauge boson predicted in this model. We emphasized that although these new contributions may be sizable, they have no direct impact since they do not contain any strong CP violating phase. Therefore, they contribute to lepton asymmetry via the interference with one loop vertex and self-energy diagrams. In this respect, a successful baryogenesis can be obtained in the resonant leptogenesis scenario where the right handed neutrinos are semi-degenerate in masses.

\section{References}

[1] R. N. Mohapatra and R. E. Marshak, Phys. Rev. Lett. 44, 1316 (1980); R. E. Marshak and R. N. Mohapatra, Phys. Lett. B 91, 222 (1980); C. Wetterich, Nucl. Phys. B 187, 343 (1981); A. Masiero, J. F. Nieves and T. Yanagida, Phys. Lett. B 116, 11 (1982); R. N. Mohapatra and G. Senjanovic, Phys. Rev. D 27, 254 (1983); R. E. Marshak and R. N. Mohapatra, S. S. Rao, W. Buchmuller, C. Greub and P. Minkowski, Phys. Lett. B 267, 395 (1991). D. G. Cerdeno, A. Dedes and T. E. J. Underwood, JHEP 0609 (2006) 067 [arXiv:hep-ph/0607157]; W. F. Chang, J. N. Ng and J. M. S. Wu, arXiv:hep-ph/0701254. F. del Aguila, M. Masip and M. Perez-Victoria, Nucl. Phys. B 456, 531 (1995) [arXiv:hep-ph/9507455]. W. Buchmuller, C. Greub and P. Minkowski, Phys. Rev. D 66, 010001 (2002).

[2] S. Khalil, arXiv:hep-ph/0611205.

[3] W. Emam and S. Khalil, arXiv:0704.1395 [hep-ph].

[4] A. G. Cohen, D. B. Kaplan and A. E. Nelson, M.B. Gavela, P. Hernandez, J. Orloff, O. Pène and C. Quimbay, Nucl. Phys. B 430, 345 (1994); Nucl. Phys. B 430, 382 (1994); A. D. Dolgov, arXiv:hep-ph/9707419.; V.A. Rubakov and M.E. Shaposhnikov, Usp. Fiz. Nauk 166 (1996) 493 [Phys. Usp. 39 (1996) 461].

[5] M. C. Chen, arXiv:hep-ph/0703087, and references therein.

[6] M. Carena, A. Daleo, B. A. Dobrescu and T. M. P. Tait, Phys. Rev. D 70, 093009 (2004); T. Appelquist, B. A. Dobrescu and A. R. Hopper, Phys. Rev. D 68, 035012 (2003). 
[7] A. Abulencia et al. [CDF Collaboration], Phys. Rev. Lett. 96, 211801 (2006) [arXiv:hep-ex/0602045].

[8] M. Altmann et al. [GNO COLLABORATION Collaboration], Phys. Lett. B 616, 174 (2005); B. Aharmim et al. [SNO Collaboration], Phys. Rev. D 72, 052010 (2005); T. Araki et al. [KamLAND Collaboration], Phys. Rev. Lett. 94, 081801 (2005); Y. Ashie et al. [Super-Kamiokande Collaboration], Phys. Rev. D 71, 112005 (2005); E. Aliu et al. [K2K Collaboration], Phys. Rev. Lett. 94, 081802 (2005); P. Adamson et al. [MINOS Collaboration], Phys. Rev. D 73, 072002 (2006); M. Ambrosio et al. [MACRO Collaboration], Eur. Phys. J. C 36, 323 (2004).

[9] R. N. Mohapatra et al., arXiv:hep-ph/0510213; R. N. Mohapatra and A. Y. Smirnov, Ann. Rev. Nucl. Part. Sci. 56 (2006) 569;A. Strumia and F. Vissani, arXiv:hep$\mathrm{ph} / 0606054$.

[10] D. N. Spergel et al. [WMAP Collaboration], Astrophys. J. Suppl. 148, 175 (2003);

M. Tegmark et al. [SDSS Collaboration], Phys. Rev. D 69, 103501 (2004).

[11] W. Buchmuller, M. Plumacherr Physics Letters B 431(1998)354362

[12] A.pilaftsis and T. E. J. Underwood, Nucl. Phys. B692 (2004) 303; Wan-lei Guo, Zhizhong Xing and Shun Zhou, hep-ph/0612033; J.Ellis, M. Radial and T. Yanagida, Phys. Lett. B546 (2002) 228; Zhi-zhong Xing and Shun Zhou, Phys. Lett. B653 (2007) 278; S. M. West, Mod.Phys.Lett. A21 (2006) 1629; T. E. J. Underwood, hep-ph/0605232.

[13] M. Fukugita and T. Yanagida, Phys. Lett. B 174, 45 (1986);

M. Plumacher, Z. Phys. C 74, 549 (1997);

W. Buchmuller and M. Plumacher, Int. J. Mod. Phys. A 15, 5047 (2000);

W. Buchmuller, P. Di Bari and M. Plumacher, New J. Phys. 6, 105 (2004);

G. F. Giudice, A. Notari, M. Raidal, A. Riotto and A. Strumia, Nucl. Phys. B 685, 89 (2004).

[14] T. Asaka, K. Hamaguchi, M. Kawasaki and T. Yanagida, Phys. Lett. B 464, 12 (1999); T. Fukuyama, T. Kikuchi and T. Osaka, JCAP 0506, 005 (2005); T. Dent, G. Lazarides and R. Ruiz de Austri, Phys. Rev. D 72, 043502 (2005); A. Mazumdar, Phys. Lett. B 580, 7 (2004); G. Panotopoulos, Phys. Lett. B 643, 279 (2006); 